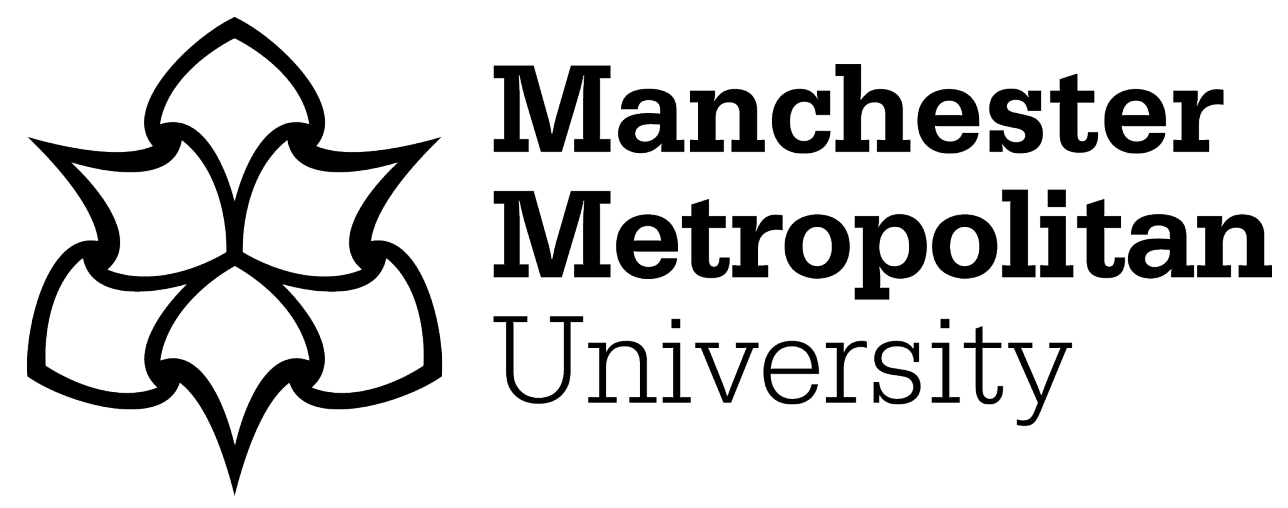

Jayes, Mark J ORCID logoORCID: https://orcid.org/0000-0002-0371-7811 and Palmer, Rebecca $L$ (2014) Stroke Research Staff's Experiences of Seeking Consent from People with Communication Difficulties: Results of a National Online Survey. Topics in Stroke Rehabilitation, 21 (5). pp. 443-451. ISSN 1074-9357

Downloaded from: https://e-space.mmu.ac.uk/624137/

Version: Accepted Version

Publisher: Taylor \& Francis

DOI: https://doi.org/10.1310/tsr2105-443

Please cite the published version 


\title{
Stroke Research Staff's Experiences of Seeking Consent from People with Communication Difficulties: Results of a National Online Survey
}

\author{
Mark J. Jayes, MSc, ${ }^{1}$ and Rebecca L. Palmer, $\mathrm{PhD}^{1,2}$ \\ ${ }^{1}$ Sheffield Teaching Hospitals NHS Foundation Trust, Sheffield, UK; ${ }^{2}$ University of Sheffield, Sheffield, UK
}

\begin{abstract}
Background: The process of obtaining informed consent from people with communication difficulties is challenging. An online survey was conducted to explore the experiences of stroke research staff in seeking consent from this population. Objectives: To identify how stroke research staff seek consent from people with communication difficulties, potential barriers to effective practice, and ways to improve practice. Methods: All research staff working for the National Institute for Health Research Stroke Research Network in England were invited to complete an online questionnaire. Data were collected anonymously between March and June 2013. Quantitative data were analyzed using descriptive statistics, and qualitative data were coded using thematic analysis. Results: Seventy-five research staff responded, corresponding to a response rate of $10 \%$. There were $97 \%$ who had sought consent from people with communication difficulties and $52 \%$ did this regularly; $65 \%$ had received training in consenting this population. Most staff were aware of appropriate methods for supporting communication needs, but only $18 \%$ regularly used accessible information and $35 \%$ regularly used augmentative communication techniques. Lack of specific training and lack of access to ethically approved materials were suggested barriers to using these methods. Respondents indicated that people with impaired communication may be excluded from the consent process because they are not eligible for inclusion in studies or because assent is obtained from third parties. Conclusions: For research staff to work more effectively with this population, study protocols need to be more inclusive of people with communication difficulties, and staff need better access to ethically approved, accessible communication resources and appropriate training. Key words: aphasia, communication, consent, stroke
\end{abstract}

$\mathrm{T}$ he exclusion of people with aphasia from stroke research has been identified as a potential threat to the establishment of robust research evidence and effective clinical interventions for stroke. ${ }^{1}$ The exclusion of this group may, in part, result from the specific challenges associated with obtaining informed consent from people with impaired communication skills. ${ }^{2}$

In England and Wales, the legal framework for obtaining consent for both research participation and treatment is provided by the Mental Capacity Act (MCA). ${ }^{3}$ In order for it to be concluded that an individual is able to provide informed consent, he or she must demonstrate the ability to understand, retain, and weigh up information that is relevant to decision making and must be able to communicate his or her decision. ${ }^{3}$ The nature of an individual's communication difficulties can make it difficult

Corresponding author: Mark J. Jayes, MSc, Speech and Language Therapy, Vickers Main Hall, Northern General Hospital, Herries Road, Sheffield, S5 7AU, UK; e-mail: mark.jayes@sth.nhs.uk for research or health care staff without specialist knowledge to accurately assess these abilities. ${ }^{4}$ The MCA requires researchers to (1) assess whether a potential research participant is able to make an informed decision about participating in a study and (2) seek assent from a consultee (eg, a relative or friend) when the assessment indicates that an individual lacks this ability. In contrast, when consent is sought for treatment and mental capacity assessment establishes that a patient lacks the capacity to make an informed decision, health care staff are required to seek the views of all significant people involved in the patient's care, including those of the patient's relatives and friends in order to make a treatment decision in the patient's best interest. ${ }^{3}$

Top Stroke Rehabil 2014;21(5):443-451

(c) 2014 Thomas Land Publishers, Inc

www.strokejournal.com

doi: $10.1310 /$ tsr2105-443 
The MCA also requires that individuals be supported to understand relevant information in whichever accessible format is most helpful to them. Information can be made more accessible to people with aphasia when it incorporates simplified language and formatting and when pictorial or photographic images are used to represent key concepts. ${ }^{5}$ In addition, augmentative communication techniques can be used to supplement or replace spoken and written language to assist people with impaired comprehension to understand information. These techniques involve the use of different communication methods, such as drawing, gesture, or pointing to words or images. ${ }^{6}$ The MCA and its Code of Practice ${ }^{7}$ do not provide the specific knowledge, skills, and practical tools that researchers would need to be able to adapt written and spoken information to make it more accessible.

Currently, there is limited evidence relating to the process of obtaining informed consent from people with communication disorders. ${ }^{8}$ Data suggest that people with aphasia tend to be excluded, either from participating in research entirely ${ }^{9}$ or from the informed consent process, because researchers tend to obtain assent for participation from others on their behalf. ${ }^{10}$ Evidence also indicates that researchers lack awareness of how impaired communication skills may affect an individual's ability to provide informed consent. ${ }^{11,12}$

This article presents the results of an online survey that was designed to investigate stroke research staff's experiences of seeking consent from people with impaired communication. The specific aims of this study were to identify (1) ways in which research staff work with people who have communication difficulties during the consent process, (2) factors that may act as barriers to staff working effectively with these people during the consent process, and (3) ways to improve research staff's practice when seeking consent from people with impaired communication.

\section{Methods}

\section{Selection of participants}

All active research staff within the National Institute of Health Research Stroke Research Network in England were eligible for inclusion in this study. The Stroke Research Network supports a range of research studies in acute care, primary care, and rehabilitation settings. These studies may involve research with greater than minimal risk (eg, drug trials). These research staff were chosen because they are likely to work with people with impaired communication, which is a common consequence of stroke. ${ }^{13}$ We asked the Communications Manager of the Stroke Research Network to publicize the study and distribute an electronic information sheet to potential participants using established electronic distribution lists. All participants who responded to the survey within a 3-month data collection period between March and June 2013 were included.

\section{Survey instrument}

The online survey design tool SurveyMonkey was used to create an electronic questionnaire. This tool enables secure, anonymous data collection and ensures confidentiality. ${ }^{14}$ The questionnaire was designed to collect quantitative and qualitative data to meet the study aims. Quantitative data were collected using multiple-choice questions, and qualitative data were collected using free text questions. Questions were created to collect the following information: (a) respondents' job roles and regional locations; (b) respondents' experience of training on how to seek consent from people with communication difficulties; (c) how respondents determine whether participants have communication difficulties; and (d) whether respondents use accessible information and augmentative communication techniques during the consent process.

The questionnaire was piloted before the study to ensure that the questions were unambiguous and generated the anticipated types of data. Five health services researchers working within the University of Sheffield were asked to complete the survey and provide feedback. Subtle changes were made to the questionnaire on the basis of this feedback.

\section{Data collection and analysis}

Participants' responses were stored anonymously and securely on the password-protected survey Web site. At the end of data collection, individual responses were transferred to a Microsoft Excel 
spreadsheet for analysis. Summaries of quantitative data were generated using the SurveyMonkey software. Quantitative data were analyzed using descriptive statistics; important findings were illustrated using response frequency and percentage tables and bar and pie charts. Qualitative data were examined and coded by the primary author (M.J.) using thematic analysis. Important themes arising from these data were reported and illustrated using sections of original data.

\section{Ethical approval}

This research was approved by the University of Sheffield School of Health and Related Research Ethics Committee.

\section{Results}

\section{Participants}

Eighty-one research staff consented to take part; 75 participants started to take the survey, and full responses were obtained from 59. Our best estimate for the total number of eligible respondents who received the survey is 720 . This number equates to the number of staff likely to be involved in recruitment to studies across the 8 regional areas of the Stroke Research Network in England. Therefore, the estimated response rate for the survey was $10 \%$. The majority of respondents were employed as research nurses (43\%) or researchers (31\%). Other respondents worked in research as part of their professional roles as doctors, nurses, or allied health professionals (Figure 1). Research staff from 9 geographical regions in England participated (Figure 2); most respondents reported working in the North West. Although the survey was designed to be sent to research staff working for the Stroke Research Network in England, 1 respondent (P70) reported working in Scotland. This respondent's data have been included in analysis, because the legislative framework governing consent in Scotland ${ }^{15}$ closely resembles the statutory requirements of the MCA. Unfortunately, the data collected do not allow us to compare characteristics of respondents to the survey with those of nonrespondents.

\section{Working with people with communication difficulties during the consent process}

Research staff were asked how frequently they seek consent from people with impaired

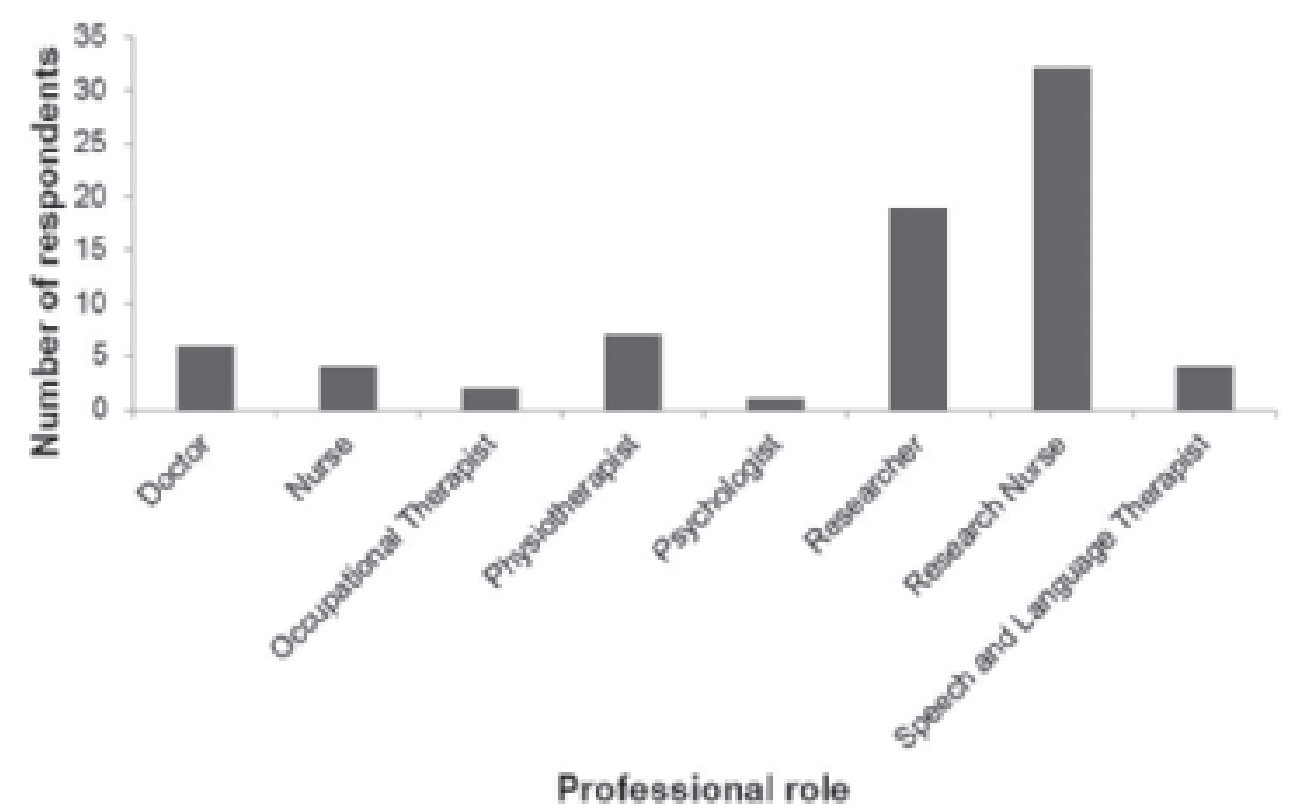

Figure 1. Distribution of respondents' professional roles $(n=75)$. 


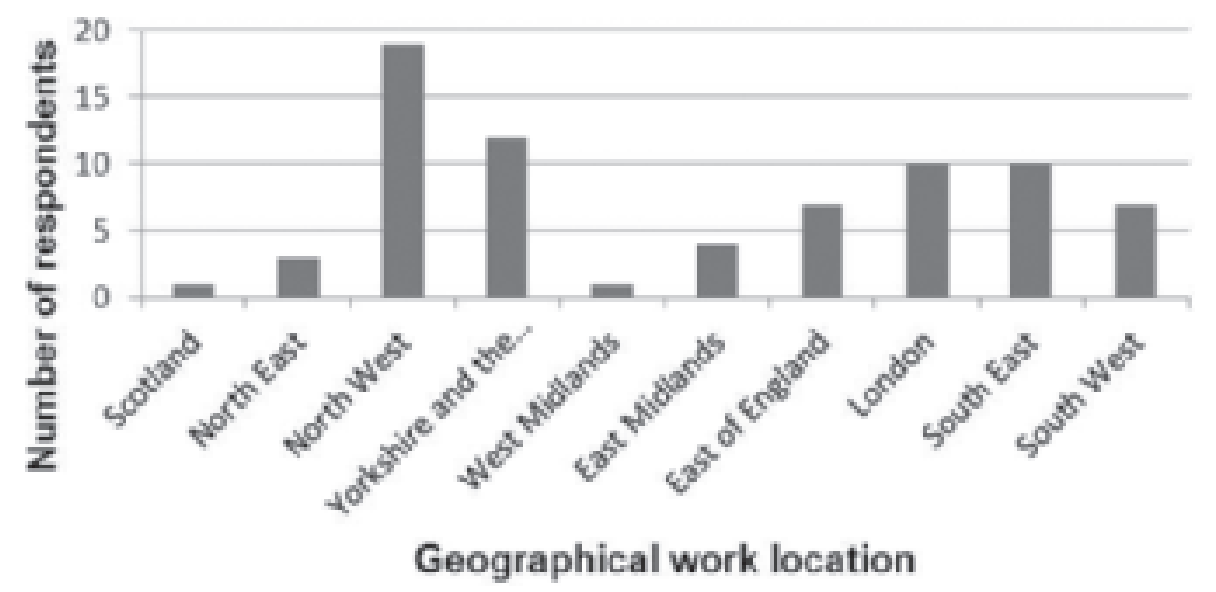

Figure 2. Distribution of respondents' work locations $(n=75)$.

communication. Their responses are shown in Table 1. Almost all respondents (73; 97\%) had experience seeking consent from people with communication difficulties.

Respondents who reported that they did not seek consent from this group regularly were asked why this was the case. Important themes were identified from their responses. The most common reason provided was that people with communication difficulties are usually not eligible for inclusion in studies (11 respondents; $32 \%)$. Another important reason was that staff tend to involve others in the consent process. Three research staff suggested they would seek assent for participation from a third party (eg, a relative) rather than seek consent from an individual with impaired communication.

Table 1. Frequency with which respondents seek consent from people with communication difficulties

\begin{tabular}{lcc}
\hline $\begin{array}{l}\text { Frequency with which } \\
\text { respondents seek } \\
\text { consent from people with } \\
\text { communication difficulties }\end{array}$ & $\begin{array}{l}\text { Frequency } \\
\text { of response }\end{array}$ & $\begin{array}{l}\text { Percentage of } \\
\text { respondents }\end{array}$ \\
\hline Never & 2 & 3 \\
Rarely & 10 & 13 \\
Sometimes & 24 & 32 \\
Regularly & 31 & 41 \\
Often & 8 & 11 \\
\hline
\end{tabular}

Two nurses reported they would collaborate with a speech and language therapist and not seek consent themselves. Additional reasons for not seeking consent regularly from this group related to the perceived level of difficulty involved in establishing whether a person with communication problems has the capacity to give informed consent and the time required to do this. Two research nurses suggested that consenting participants with impaired communication is particularly challenging because of the need to achieve timely recruitment to a study:

Sometimes the window of opportunity to take consent within the time limits given in the study's protocol is so small that there isn't time to explore other means of communicating and then patients may have been able to consent for themselves but are lost to the study. [P26]

I find I often go to [the] relative because of time pressures whereas the patient if [s/he] has capacity should have the chance to consent for themselves. [P80]

Ways of supporting people with communication needs during the consent process

\section{Identifying communication difficulties}

Research staff reported using a range of methods to determine whether people have communication difficulties (Table 2). Nine respondents reported using either a communication or cognitive screening tool; these included the Frenchay Aphasia Screening Test, ${ }^{16}$ the Sheffield Screening Test for Acquired Language Disorders, ${ }^{17}$ and the Montreal Cognitive Assessment. ${ }^{18}$ 
Table 2. Methods used to identify communication difficulties

\begin{tabular}{lcc}
\hline $\begin{array}{l}\text { Methods research staff use to identify communication difficulties } \\
\text { (including themes identified from qualitative responses) }\end{array}$ & $\begin{array}{c}\text { Frequency } \\
\text { of response }\end{array}$ & $\begin{array}{c}\text { Percentage of } \\
\text { respondents }\end{array}$ \\
\hline Talking to the participant & 52 & 91 \\
Asking participant questions to check that he/she understands & 48 & 84 \\
Seeking advice from speech and language therapists & 43 & 75 \\
Seeking advice from the multidisciplinary clinical team/reading & 22 & 39 \\
medical notes & 7 & 12 \\
Using a communication screening tool & 5 & 9 \\
Consulting relatives & 2 & 4 \\
Using a cognitive screening tool & & \\
\hline
\end{tabular}

\section{Accessible information}

Research staff were asked how often they use accessible information (ie, information that is adapted to make it simpler to understand) to support people with communication difficulties during the consent process. Their responses are shown in Table 3. Thirty-eight respondents (68\%) reported using accessible information.

Table 3 shows that almost a third of respondents reported that they do not use accessible information. The primary reason research staff gave for this was that accessible information formats are not made available for staff to use $(12 ; 67 \%)$. Two research nurses commented:

To my knowledge none of the studies I work on have included the provision of such information. [P21]

The ethically approved versions are not aphasia friendly and we can't change the approved versions. [P46]

Respondents suggested that they would find it easier to use accessible information if it were more readily available, particularly if it were provided at the start of a research study. Staff also indicated that they would be able to create and use accessible information more easily if they were given more

Table 3. Frequency with which staff use accessible information

\begin{tabular}{lcc}
\hline $\begin{array}{l}\text { Frequency with which } \\
\text { staff use accessible } \\
\text { information }\end{array}$ & $\begin{array}{l}\text { Frequency } \\
\text { of response }\end{array}$ & $\begin{array}{l}\text { Percentage of } \\
\text { respondents }\end{array}$ \\
\hline Not used & 18 & 32 \\
Used occasionally & 16 & 29 \\
Used sometimes & 12 & 21 \\
Used regularly & 10 & 18 \\
\hline
\end{tabular}

training and/or had access to resources such as handbooks, guidelines, or a dedicated Web site.

\section{Augmentative communication techniques}

Research staff were asked how frequently they use augmentative communication techniques (such as gesture, mime, or drawing) to support people with communication difficulties during the consent process. Their responses are shown in Table 4. Forty-five respondents (80\%) reported using these techniques. These individuals described using a number of techniques, including gesture, pointing to words and pictures, alphabet charts, and drawing.

Respondents provided a number of reasons for not using augmentative techniques; these tended to be similar to the reasons provided for not using accessible information. Research staff reported that they lack appropriate training in using these techniques or do not have access to ethically approved resources. They also suggested that these techniques are not used because people with impaired communication are usually not eligible for inclusion in studies or because local practice is to take assent from a third party rather than

Table 4. Frequency with which staff use augmentative communication techniques

\begin{tabular}{lcc}
\hline $\begin{array}{l}\text { Frequency with which } \\
\text { staff use augmentative } \\
\text { communication techniques }\end{array}$ & $\begin{array}{l}\text { Frequency } \\
\text { of response }\end{array}$ & $\begin{array}{l}\text { Percentage of } \\
\text { respondents }\end{array}$ \\
\hline Not used & 11 & 20 \\
Used occasionally & 18 & 32 \\
Used sometimes & 13 & 23 \\
Used regularly & 14 & 25 \\
\hline
\end{tabular}


seek consent from a person with communication difficulties. Again, research staff reported they would find it easier to use these techniques if they had better access to training and guidance and if they were given sufficient time.

\section{Additional methods used to support people with impaired communication}

Eleven respondents (20\%) reported using additional methods to support people with communication difficulties during the informed consent process. The most common method reported was involving the individual's family or a significant other in the consent process ( 9 ; 82\%). Respondents' comments suggest that the involvement of a third party can serve various purposes. A family member or carer can provide useful background information on the individual's communication skills and practical support for the individual during the consent process. A family member or carer can also advocate on the individual's behalf and provide assent to participate in a study, if appropriate. Two staff reported that they seek clinical advice (eg, from speech and language therapists) to help them support people with communication needs. Other respondents said they try to give people sufficient time to communicate during the consent process and may discuss consent-related issues on more than one occasion. A speech and language therapist reported making attempts to adjust the environment to facilitate communication about consent.

\section{Experience of training in seeking consent from people with communication difficulties}

Forty-five respondents (65\%) had received training on consenting people with impaired communication. Forty of these respondents (93\%) found the training useful. Thirteen respondents (34\%) reported that training was most useful when it included a practical component, such as a communication activity involving people with the language disorder aphasia. Respondents also valued gaining knowledge in specific areas, including mental capacity assessment for people with impaired communication and appropriate methods to use to support people's communication needs.

Twenty-five research staff (66\%) reported that the training they had received had led to changes in their practice when seeking consent from this group. Twelve of these respondents (48\%) said that they now use strategies, tools, and other resources to make communication more accessible to participants during the consent process. One researcher commented:

[I] use props as a way to demonstrate the research more clearly or to facilitate [people with communication difficulties] in expressing their understanding. [P60]

Four respondents suggested that training had helped them to make their recruitment to studies more inclusive of people with impaired communication. A research nurse (P46) commented: "I try and involve patients as much as possible in the consent process." Respondents also reported other changes to their practice after training, including (a) improved mental capacity assessment, (b) ability to tailor the consent process to individual communication needs, (c) enhanced documentation, and (d) greater involvement of others to support the consent process (eg, a participant's family, a speech and language therapist).

Thirty-three respondents (56\%) reported that they would appreciate more training on how to seek consent from people with communication difficulties. Staff identified a number of potential training topics; major themes identified in their responses are shown in Table 5.

Table 5. Areas for additional training identified by research staff

\begin{tabular}{lcc}
\hline $\begin{array}{l}\text { Areas for additional training identified by staff } \\
\text { (themes identified from qualitative responses) }\end{array}$ & $\begin{array}{c}\text { Frequency } \\
\text { of response }\end{array}$ & $\begin{array}{c}\text { Percentage of } \\
\text { respondents }\end{array}$ \\
\hline Communication techniques/strategies/tools/aids & 13 & 42 \\
Legislation & 3 & 10 \\
Aphasia/patients with aphasia & 2 & 6 \\
Communication assessments/screening tools & 2 & 6 \\
Development of information materials for participants & 1 & 3 \\
\hline
\end{tabular}




\section{Discussion}

The results of the survey suggest that almost all research staff who responded have some experience of seeking consent from participants with impaired communication; more than half the respondents stated that they do this regularly or often, as part of their professional roles. The data indicate that most staff are aware of a variety of appropriate methods to use to identify participants with communication difficulties and support their needs during the consent process.

However, participants' responses suggest a number of factors that may represent barriers to staff seeking consent from participants with communication difficulties. Seeking consent from this population is perceived by some to be both a difficult and time-consuming activity, which may not always be compatible with the need to achieve timely recruitment to satisfy the requirements of study protocols. Although staff appear to be aware of supportive methods such as accessible information or augmentative communication techniques, the majority report they do not use these regularly. These resources may not always be made available to staff or might not be ethically approved for use in studies. Even when such resources are made available, staff may not be trained to use them. The data collected do not enable us to speculate whether staff would approach the process of seeking consent from participants with communication difficulties differently in situations in which a study involved greater than minimum risk.

These factors all affect the ability of research staff to include and engage people with impaired communication in the consent process effectively and could mean that staff decide not to seek consent from this group at all. It is unclear from the data what proportion of people with communication difficulties might be excluded from studies because their consent to participate cannot be obtained. A number of respondents stated that they are likely to seek assent for participation from a third party in cases in which a potential study recruit has communication difficulties. It is worth reasserting that assent should only be sought once it has been established that a participant is unable to provide informed consent; the assessment of mental capacity required to establish this should always include active measures to support the individual to understand information relevant to decision making and communicate a decision. ${ }^{7}$ Failure to adhere to this legal process is unethical because it means people with communication difficulties are being denied the right to make decisions for themselves. ${ }^{3}$

The most common reason respondents provided for not seeking consent from people with impaired communication was that these people would not be eligible for inclusion in studies. This finding is consistent with previous evidence that people with communication difficulties have been excluded from stroke research. ${ }^{9}$ Excluding people from participating on the basis of their communication difficulties threatens both the ethical conduct of studies and the generalizability of their findings ${ }^{2}$; furthermore, there is a clear risk that the underrepresentation of this clinical group within the evidence base will lead to parallel inequalities in treatment planning and care provision. ${ }^{1}$

The inclusion of people with communication disorders within the informed consent process, and within studies in general, can be facilitated if researchers ensure that accessible communication materials are incorporated into the initial planning and design of studies. In addition, study protocols should enable research staff to take sufficient time to use accessible materials and augmentative communication methods. Applications to ethics committees need to include information about the use of such materials and methods to ensure that appropriate permissions are obtained before the study begins. This would address one of the main barriers to using accessible communication methods cited by research staff in this study.

The survey results demonstrate the potential for regular, targeted training to empower research staff to make the consent process more accessible to and inclusive of people with communication difficulties. Training may be most beneficial when it involves opportunities to practice skills and techniques directly with people with communication impairments. Previous studies suggest that the involvement of service users in the delivery of health care 
training can enhance trainees' communication skills and also their ability to adopt an empathic, person-centered approach toward service users. ${ }^{19}$

It is important to acknowledge several limitations to this study. The sample size was relatively small, which has implications for the generalizability of the findings. Furthermore, because the Stroke Research Network is committed to increasing the participation of people with impaired communication within research, the respondents to this survey may possess increased levels of training, knowledge, and skills in working with this patient group, compared with other research staff. A limitation of the data collection method chosen was that the authors were not able to explore certain responses in greater detail or verify the meaning of ambiguous or incomplete responses. It is recommended that future studies use focus group or individual interviews to enable a more thorough investigation.

Also, it is important to acknowledge that the survey asked respondents about their knowledge and skills in seeking consent from people with impaired communication but did not directly test this knowledge or observe these skills in practice. Therefore, the data do not allow us to determine whether the respondents are working competently. Future studies could use survey, interview, or ethnographic methods to overcome this limitation. These methods have been used previously within the field of mental capacity assessment. ${ }^{20-22}$

\section{REFERENCES}

1. Brady MC, Fredrick A, Williams B. People with aphasia: Capacity to consent, research participation and intervention inequalities. Int J Stroke. 2013;8:193-196.

2. Jayes $M$, Palmer R. Initial evaluation of the Consent Support Tool: A structured procedure to facilitate the inclusion and engagement of people with aphasia in the informed consent process. Int J Speech Lang Pathol. 2014;16(2): 159-168.

3. Office of Public Sector Information. Mental Capacity Act. London: Office of Public Sector Information; 2005.

\section{Conclusion}

In summary, this study indicates that people with communication difficulties remain at risk of being excluded from the informed consent process and from participating in research generally. Although most stroke research staff in England have experience seeking consent from people with impaired communication, it appears that only a minority are able to use appropriate methods regularly to include and support these people within the process. In order for research staff to work more effectively with this population, it is suggested that study protocols need to facilitate greater inclusion of people with communication difficulties, and staff need to have better access to ethically approved, accessible communication resources and appropriate training.

\section{Acknowledgments}

The study was supported by the National Institute for Health Research (NIHR) Collaboration for Leadership in Applied Health Research and Care for South Yorkshire (CLAHRC SY). CLAHRC SY acknowledges funding from the National Institute for Health Research. The views and opinions expressed are those of the authors and not necessarily those of the National Health Service, the NIHR, or the Department of Health. CLAHRC SY would also like to acknowledge the participation and resources of their partner organizations. Further details can be found at www.clahrc-sy.nihr.ac.uk.

The authors report no conflicts of interest.

4. Ferguson A, Duffield G, Worrall L. Legal decisionmaking by people with aphasia: Critical incidents for speech pathologists. Int I Lang Commun Disord. 2010;45(2):244-258.

5. Rose TA, Worrall LE, Hickson LM, Hoffmann TC. Aphasia friendly written health information: Content and design characteristics. Int / Speech Lang Pathol. 2011;13(4):335-347.

6. Dalemans R, Wade D, van der Heuvel WJA, de Witte LP. Facilitating the participation of people with aphasia in research: A description of strategies. Clin Rehabil. 2009;23:948-959. 
7. Department of Constitutional Affairs. Mental Capacity Act Code of Practice. London: Author; 2007.

8. Carling-Rowland A, Wahl J. The evaluation of capacity to make admission decisions: Is it a fair process for individuals with communication barriers? Med Law Int. 2010;10(3):171-190.

9. Townend E, Brady M, McLaughlan K. Exclusion and inclusion criteria for people with aphasia in studies of depression following stroke: A systematic review and future recommendations. Neuroepidemiology. 2007;29:1-17.

10. Stein J, Brady Wagner L. Is informed consent a "yes or no" response? Enhancing the shared decisionmaking process for persons with aphasia. Top Stroke Rehabil. 2006;13(4):42-46.

11. Penn C, Frankel T, Watermeyer J, Müller M. Informed consent and aphasia: Evidence of pitfalls in the process. Aphasiology. 2009;23(1): 3-32.

12. Carlsson E, Paterson BL, Scott-Findlay S, Ehnfors M, Ehrenberg A. Methodological issues in interviews involving people with communication impairments after acquired brain damage. Qual Health Res. 2007;17(10):1361-1371.

13. Stroke Association. Communication problems after stroke. http://www.stroke.org.uk/factsheet/ communication-problems-after-stroke. Accessed June 8, 2013.

14. Buchanan EA, Hvizdak EE. Online survey tools: Ethical and methodological concerns of human research ethics committees. I Empir Res Hum Res Ethics. 2009;4(2):37-48.

15. The Stationary Office. Adults with Incapacity (Scotland) Act. London: Author; 2000.

16. Enderby P, Wood V, Wade D. Frenchay Aphasia Screening Test. 2nd ed. Chichester: WileyBlackwell; 2006.

17. Syder D, Body R, Parker M, Boddy M. Sheffield Screening Test for Acquired Language Disorders. Windsor: NFER Nelson; 1993.

18. Nasreddine ZS, Phillips NA, Bédirian V, et al. The Montreal Cognitive Assessment (MoCA): A brief screening tool for mild cognitive impairment. / Am Geriatr Soc. 2005;53:695-699.

19. Repper J, Breeze J. User and carer involvement in the training and education of health professionals: A review of the literature. Int I Nurs Stud. 2007;44(3);511-519.

20. Evans K, Warner J, Jackson E. How much do emergency healthcare workers know about capacity and consent? Emerg Med J. 2007;24:391-393.

21. Redley M, Keeley H, Clare I, Hinds D, Luke L, Holland A. Respecting patient autonomy: Understanding the impact on NHS hospital in-patients of legislation and guidance relating to patient capacity and consent. I Health Serv Res Policy. 2011;16(1):13-20.

22. Wilner $P$, Jenkins $R$, Rees $P$, Griffiths VJ, John E. Knowledge of mental capacity issues in community teams for adults with learning disabilities. J Appl Res Intellect Disabil. 2011;24:159-171. 